\title{
Thromboprophylaxis in Head and Neck Microvascular Reconstruction
}

\author{
Manoj Abraham, MD ${ }^{1}$ Arvind Badhey, MD² Shirley Hu, MD² Sameep Kadakia, MD $^{2}$ \\ J. K. Rasamny, MD ${ }^{1}$ Augustine Moscatello, MD ${ }^{1}$ Yadranko Ducic, MD 3
}

${ }^{1}$ Department of Otolaryngology, New York Medical College, Valhalla, New York

2 Department of Otolaryngology-Head and Neck Surgery, New York Eye and Ear Infirmary of Mount Sinai, New York, New York

${ }^{3}$ Department of Otolaryngology, Otolaryngology and Facial Plastic

Surgery Associates, Fort Worth, Texas

Craniomaxillofac Trauma Reconstruction 2018;11:85-95

\begin{abstract}
Address for correspondence Sameep Kadakia, MD, Department of Otolaryngology-Head and Neck Surgery, New York Eye and Ear Infirmary of Mount Sinai, 310 East 14th St 6th Floor, New York, NY 10003 (e-mail: sameep8779@gmail.com).
\end{abstract}

\begin{abstract}
Keywords

- anticoagulation

- microvascular surgery

- thromboprophylaxis

- head and neck surgery

Head and neck patients undergoing microvascular reconstruction are at high risk for thromboembolism. While the prevention of thromboembolism has become an essential aspect of care, within the field of microsurgery, concern for anastomotic complications have hindered the creation of an accepted regimen. The aim of this review was to evaluate the risks and benefits of prophylactic agents for thromboprophylaxis. A literature search was conducted in MEDLINE, Cochrane Library, and PubMed/NCBI databases. Articles discussing thromboprophylaxis in otolaryngology, head and neck surgery, or microvascular reconstruction were considered in the review from the past 30 years. The majority of patients undergoing microvascular surgery have multiple risk factors for thrombus formation. Several consensus guidelines exist for the prophylaxis in patients who are critically ill, undergoing surgery, or with malignancy. Significant evidence supports the routine use of mechanical means, such as early mobilization and pneumatic compression along with subcutaneous heparin. Low-molecular-weight heparin is also frequently utilized, although results are largely divided. Data on aspirin remain equivocal. Studies on microvascular failure and flap loss have demonstrated little to no association with chemoprophylaxis. The evidence for postoperative thromboprophylaxis regimens in patients undergoing head and neck free tissue transfer is variable. Multiple studies have supported the use of unfractionated heparin or low-molecular-weight heparin. There appears to be an expert consensus for the combined use of mechanical prophylactic methods and chemical prophylaxis. Prospective randomized trials are required to validate the most effective combination of chemoprophylaxis agents.
\end{abstract}

Postsurgical head and neck cancer (HNC) patients are at increased risk of venous thromboembolism (VTE) and its sequelae, pulmonary embolism (PE). ${ }^{1}$ These patients exhibit many of the major risk factors for VTE, including advanced age, obesity, pulmonary disease, immobility, malignancy, and smoking, and alcohol use. ${ }^{2}$ The incidence of deep vein thrombosis (DVT) in patients with HNC following surgery ranges from 0.07 to $26.3 \%$, although these rates are derived received

April 23, 2017

accepted after revision

June 17, 2017

published online

October 31, 2017
Copyright $\odot 2018$ by Thieme Medical Publishers, Inc., 333 Seventh Avenue, New York, NY 10001, USA. Tel: +1(212) 584-4662.
DOI https://doi.org/ 10.1055/s-0037-1607068. ISSN 1943-3875. 
from retrospective studies and may underestimate true values. $^{3-9}$ In patients undergoing microvascular reconstructive surgery, the risk of VTE is further compounded.

The role of chemoprophylaxis with anticoagulants in patients undergoing microvascular reconstruction is widely debated, as bleeding complications can lead to failure of microvascular reconstruction. Other possible adverse sequelae include airway compromise and wound complications. ${ }^{10}$ These risks, along with the perception of low DVT incidence in this population, have been identified as factors contributing to the controversy of routine chemoprophylaxis following microvascular reconstruction of the head and neck.

The prophylactic treatment of DVT and PE has received sparse attention in the otolaryngology literature. There is a paucity of randomized data to guide a standard approach to DVT prophylaxis in patients undergoing free tissue transfer, and management varies widely across institutions and surgeons.

This report is the first critical review of the utility of thromboprophylaxis options in head and neck microvascular reconstruction, in an attempt to develop evidence-based recommendations for thromboprophylaxis. A thorough review of the utility of each agent with respect to thromboprophylaxis and flap survival is presented, followed by suggested recommendations derived from the authors' findings (-Table 1 ).

\section{Methods}

We aimed to identify all full-text, peer-reviewed publications pertaining to DVT prophylaxis in otolaryngology or head and neck surgery. The searches were conducted in the Ovid MEDLINE, Cochrane Library, and Google Scholar databases with no language or regional restrictions. The following keywords were used: venous thrombosis, pulmonary embolism, thromboprophylaxis, mechanical, pneumatic compression, ambulation, mobilization, aspirin, heparin, low-molecular-weight heparin, dextran, and warfarin. Results were combined with the terms otolaryngology and head and neck to retrieve articles. All articles addressing thromboprophylaxis in otolaryngology or head and neck surgery were included in the review. The reference lists of these articles were then inspected and any other pertinent publications were added. Where applicable, a search strategy was employed to identify articles discussing thromboprophylaxis in other subspecialties, such as plastic surgery and orthopaedic surgery.

\section{Results}

\section{Mechanical Methods}

Mechanical methods for thromboprophylaxis include intermittent pneumatic compression (IPC), graduated compression stockings, and venous foot pumps, which have all been demonstrated as safe and effective. ${ }^{11}$ These devices counteract stasis, one of the three components of Virchow's triad. While multiple authors recommend mechanical means as part of a larger prophylaxis protocol when a patient is at increased risk or as single modality when a patient is considered low risk, ${ }^{3,4,6,8,9,12-15}$ these studies are limited to retrospective and descriptive reviews that do not critically analyze outcomes in microvascular reconstruction.

In an extensive series of systematic reviews addressing thromboprophylaxis in surgical patients, Roderick et al ${ }^{16}$ determined that mechanical methods reduced the risk of DVT by approximately $50 \%$ and the risk of PE by $40 \%$. In a recent survey of head and neck cancer surgeons by Garritano and Andrews, ${ }^{12}$ IPC was reported as the most common form of prophylaxis in all settings (preoperatively, intraoperatively, and postoperatively). IPC has been shown to decrease the incidence of DVT in patients undergoing head and neck surgical procedures by 60 to $75 \% .^{8,17}$ In a retrospective analysis of 12,805 head and neck operations, Moreano et $\mathrm{al}^{8}$ identified IPC as one of only two statistically significant independent risk factors for the prevention of DVT. Of note, there were no instances of DVT in patients undergoing microvascular reconstruction in this study. Chen et $\mathrm{al}^{4}$ revealed similarly low rates of DVT in their study; all patients had preoperative and postoperative IPC until ambulation and received postoperative anticoagulation with aspirin and LMWH. Another retrospective cohort study of 134 patients who underwent head and neck microvascular reconstruction, however, showed no association between the use of IPC after surgery and reduced risk of DVT. ${ }^{9}$ Importantly, professional groups such as the American College of Chest Physicians (ACCP) and American Society of Clinical Oncology have made official recommendations for DVT prophylaxis that include mechanical therapy. For patients with cancer and an additional risk for DVT, the standard is LMWH in conjunction with mechanical measures. ${ }^{11}$

While data on postoperative mobilization in patients undergoing major head and neck resection with microvascular reconstruction are limited, the expert consensus is that early monitored mobilization, unless contraindicated (i.e., fibular free flap), should be a key component in a comprehensive thromboprophylactic protocol. Traditionally, concern over vessel thrombosis from vessel kinking due to neck movement resulted in delayed patient mobilization, but there has been no evidence to support or disprove the theory that postoperative mobility affects flap outcomes. ${ }^{18}$ A single retrospective cohort study of patients undergoing microvascular reconstruction ${ }^{19}$ found that earlier ambulation did not increase flap failure rates and was associated with fewer pulmonary complications. Several authors recommend mobilizing the patient as early as the second postoperative day. ${ }^{3,7,8}$ No studies, however, have shown that time to ambulation predicts the presence of DVT. ${ }^{5,9}$

\section{Aspirin}

The primary role of aspirin in hemostasis is to impair platelet aggregation through the inhibition of thromboxane A2 synthesis and reduce thrombus formation on the surface of a damaged arterial wall. ${ }^{20}$ Aspirin's effect on venous thrombosis is secondary, as the fibrin-rich red clot formation in venous thrombosis is thought to be driven by the clotting pathway. ${ }^{21}$ However, there is growing evidence that aspirin exerts other antithrombotic effects, including decreasing thrombin generation, attenuating thrombin-mediated coagulant reactions, and increasing fibrin clot permeability and 


\begin{tabular}{|c|c|c|c|c|c|c|c|c|c|}
\hline 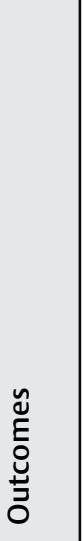 & 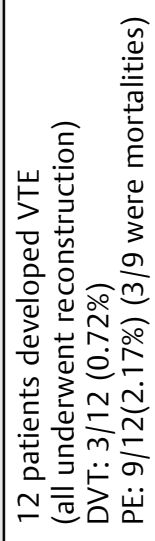 & 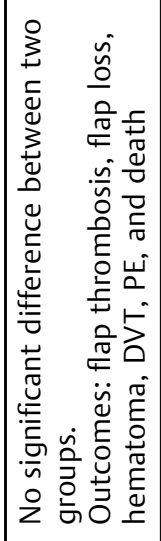 & 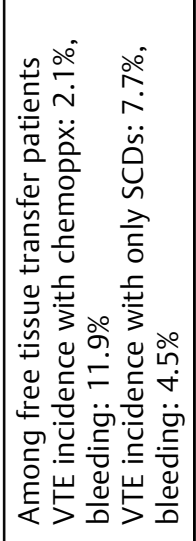 & 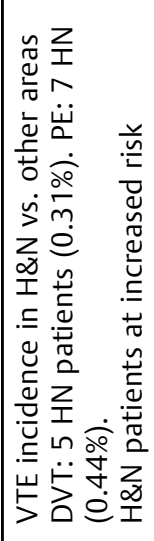 & 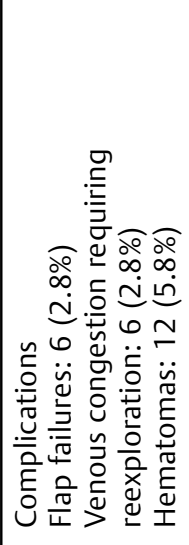 & 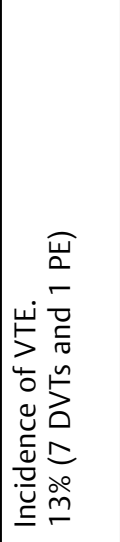 & 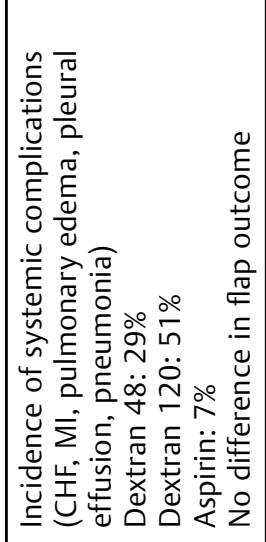 & 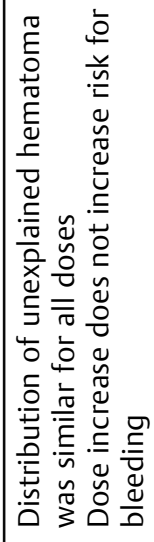 & 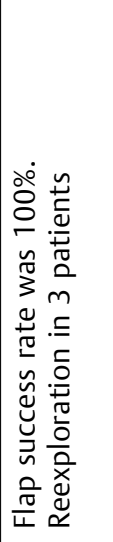 \\
\hline 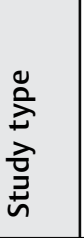 & 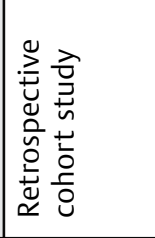 & 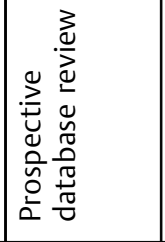 & 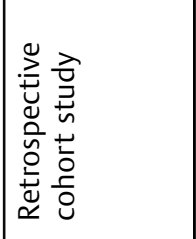 & 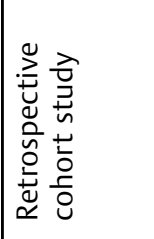 & 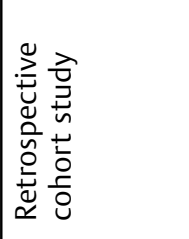 & 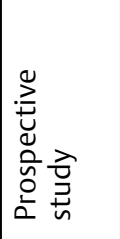 & 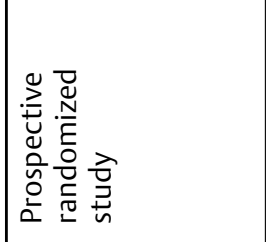 & 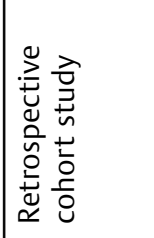 & 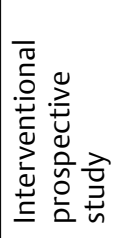 \\
\hline 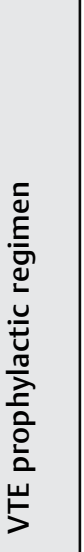 & 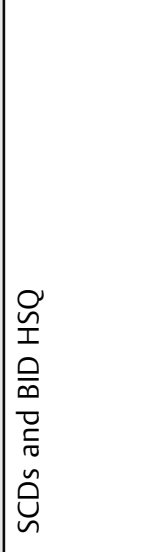 & 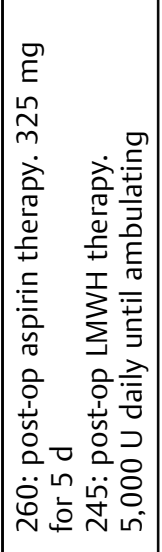 & 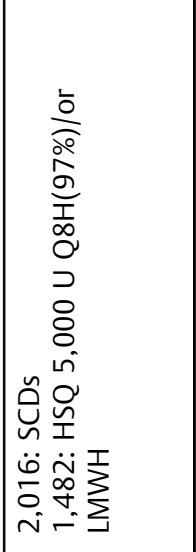 & 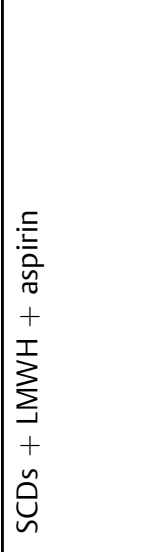 & 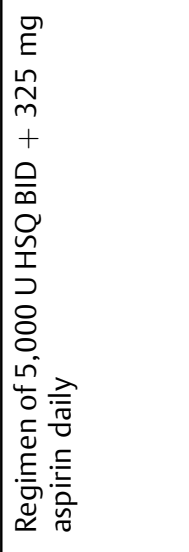 & 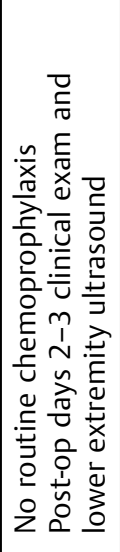 & 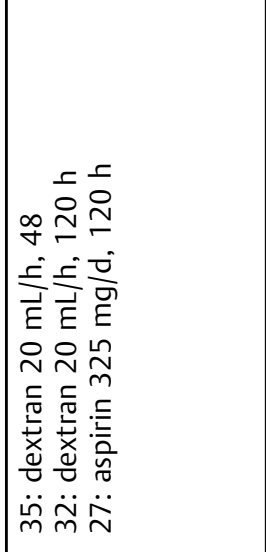 & 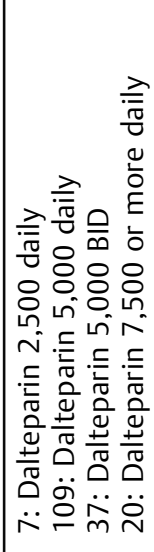 & 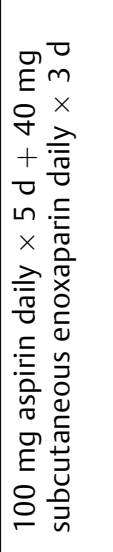 \\
\hline 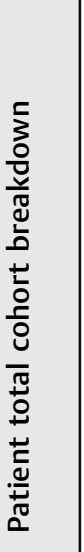 & 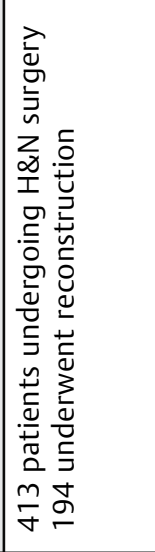 & 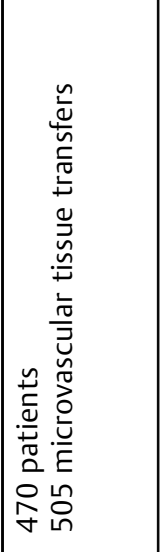 & 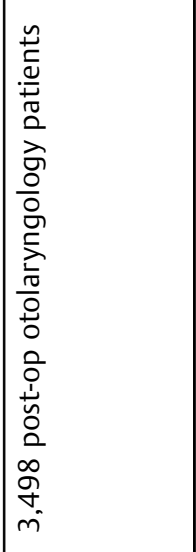 & 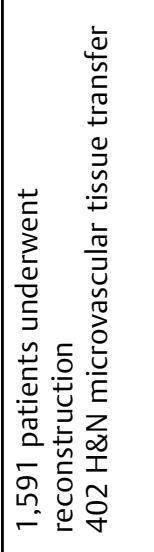 & 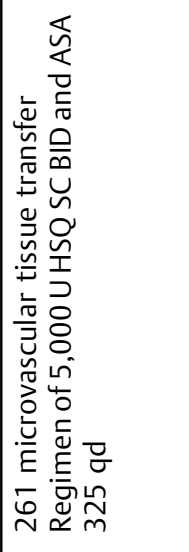 & 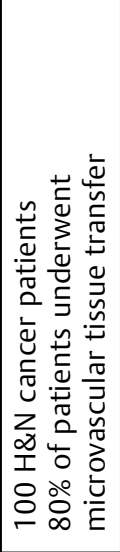 & 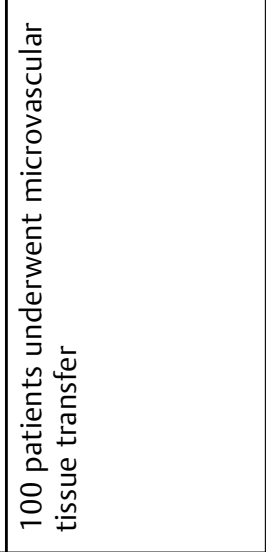 & 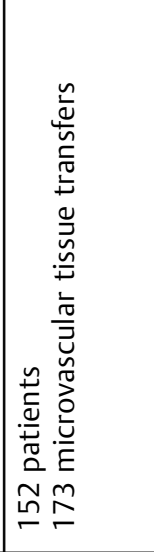 & 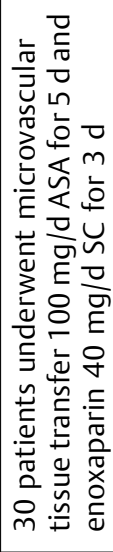 \\
\hline 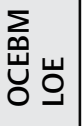 & $\hat{m}$ & $\hat{\sim}$ & $\hat{m}$ & $\hat{m}$ & $\hat{m}$ & $\vec{\sim}$ & mे & $\hat{m}$ & $\vec{\sim}$ \\
\hline 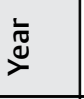 & $\frac{\ln }{\stackrel{D}{N}}$ & ì & $\stackrel{\nabla}{\stackrel{D}{N}}$ & 㐫 & 号 & $\frac{m}{i}$ & 弚 & $\frac{m}{\sim}$ & $\frac{0}{\sim}$ \\
\hline 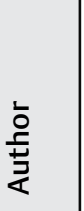 & $\begin{array}{l}\frac{m}{\pi} \\
\frac{\pi}{\omega} \\
\frac{\bar{\sigma}}{\bar{\alpha}}\end{array}$ & 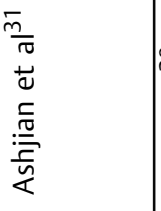 & $\begin{array}{l}\frac{\partial}{\sqrt{0}} \\
\frac{0}{0} \\
\frac{\pi}{\sqrt{0}} \\
\infty\end{array}$ & 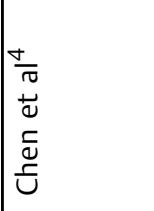 & 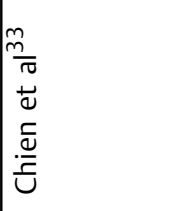 & 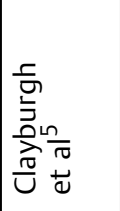 & 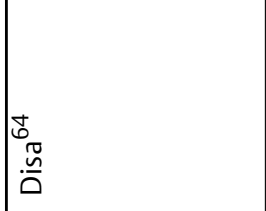 & $\begin{array}{l}\frac{\pi}{\pi} \\
\frac{\pi}{0} \\
\frac{\pi}{\omega} \\
\frac{\pi}{\omega}\end{array}$ & 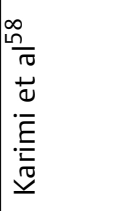 \\
\hline
\end{tabular}



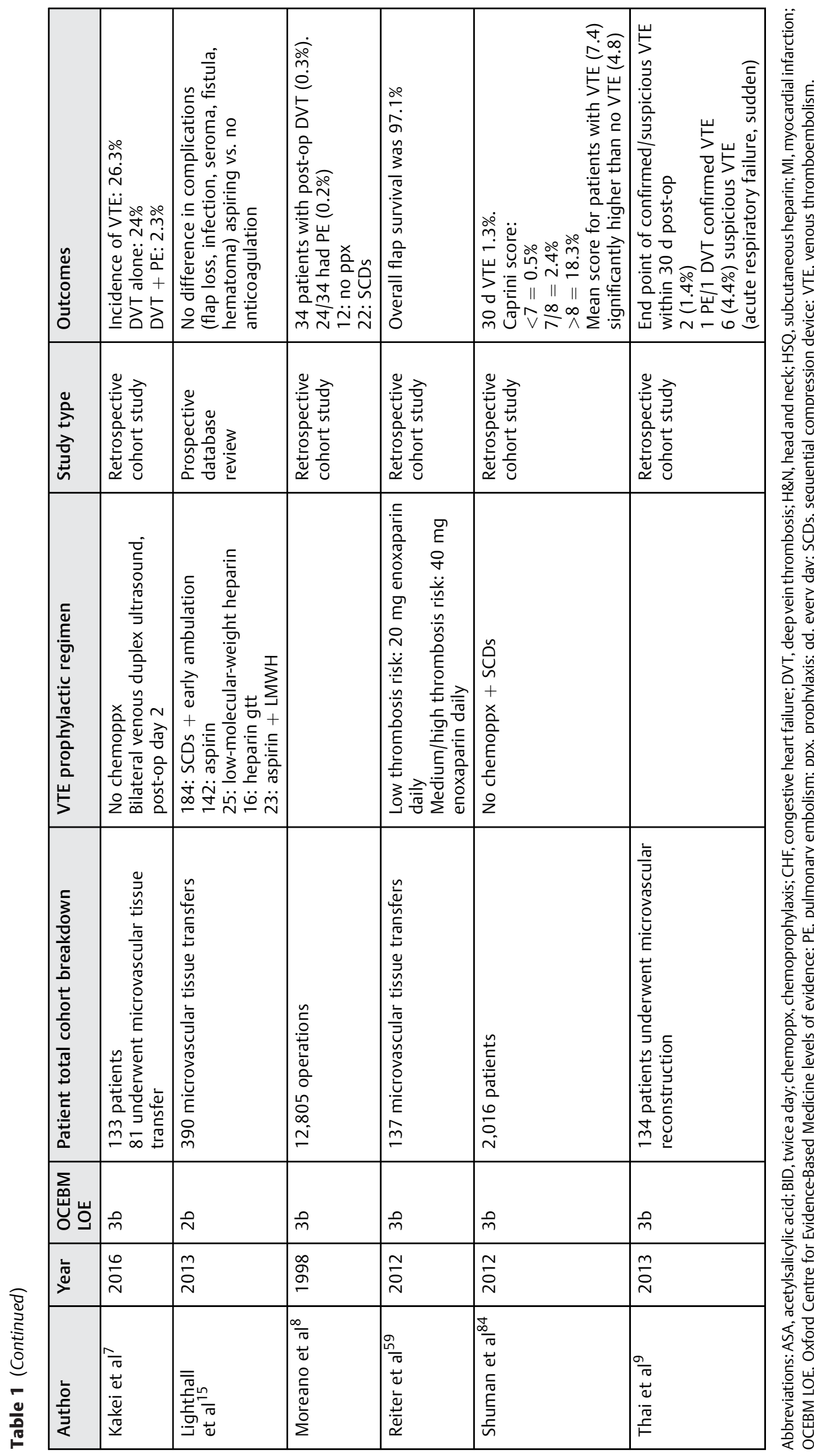
enhanced clot lysis. ${ }^{20}$ Murine models have also shown that platelets may be an important component of early DVT. Two trials, the Warfarin and Aspirin (WARFASA) study ${ }^{22}$ and the Aspirin to Prevent Recurrent Venous Thromboembolism (ASPIRE) study, ${ }^{23}$ found a decrease in recurrent VTE with a low risk of major bleeding in patients with idiopathic VTE. ${ }^{21}$

Despite this, few studies have evaluated the direct impact of aspirin on DVT outcomes in patients undergoing head and neck reconstruction. Authors more frequently describe its utilization in preventing microvascular thrombosis. Studies have shown that a platelet thrombus can occlude even a technically perfect anastomosis, ${ }^{24}$ and thus antiplatelet therapy may play a preventive role. ${ }^{25,26}$

In a survey of academic otolaryngology departments, Spiegel and Polat ${ }^{27}$ reported that $76.5 \%$ of microvascular surgeons were using aspirin, although no delineation was made whether prevention of anastomotic thrombosis or VTE was the goal. In a prospective study of 115 patients undergoing head and neck microvascular reconstruction, Blackwell reported no incidence of DVT or PE with the use of mechanical thromboprophylaxis and postoperative lowdose aspirin as the only chemoprophylactic agent. ${ }^{28}$ However, the ACCP does not recommend using aspirin alone as thromboprophylaxis against DVT in any patient population, primarily because more effective agents are available, such as unfractionated heparin (UFH) and $\mathrm{LMWH}^{2}$

Both a recent meta-analysis of the efficacy of antithrombotic regimens in microsurgery and a retrospective study of 3,498 patients undergoing otolaryngologic surgery concluded that aspirin increases the risk of hematoma significantly. ${ }^{29,30}$ Other studies have demonstrated no such increase $e^{15,28,31}$ when comparing aspirin to no prophylaxis. ${ }^{22,32}$ Chien et al $^{33}$ found similar hematoma rates between the use of aspirin and HSQ and other anticoagulation agents in a retrospective chart review of 261 patients undergoing free tissue transfer, although this comparison was not made with an internal arm. In a retrospective study of 470 microvascular patients, Ashjian et $\mathrm{al}^{31}$ found no difference in complication rates, including DVT and microvascular thrombosis, between patients treated with postoperative aspirin and those treated with LMWH. The concurrent use of aspirin and another anticoagulant has been found to be significantly associated with increased bleeding complications, potentially due to the simultaneous blockage of both the cellular, platelet-mediated clotting mechanism and the humoral clotting cascade. ${ }^{10,29,32}$

Importantly, aspirin has not been shown to be superior to other regimens or effective as a single prophylaxis drug for flap survival. ${ }^{15,33}$ One study evaluating microvascular reconstructive surgery without antithrombotic agents showed no flap failure secondary to thrombosis and concluded that the use of chemoprophylaxis alone does not seem to significantly affect the patency of microvascular structures. ${ }^{34}$

\section{Unfractionated Heparin Formulations}

UFH is a polyglycosaminoglycan that inhibits the coagulation cascade by inactivating downstream coagulation factors and platelet aggregation, primarily through increased antithrombin III antiprotease activity. Its effect is measured clini- cally with activated partial thromboplastin time, but the dose-response relationship can be unpredictable, requiring high-dose titration. ${ }^{35,36}$

\section{Subcutaneous Heparin}

Recent departmental surveys in otolaryngology have demonstrated that $26.5 \%$ of surgeons use heparin, typically postoperatively in the subcutaneous form. ${ }^{27}$ In other fields of microsurgery, subcutaneous heparin (HSQ) is the most commonly used anticoagulant in clinical practice. ${ }^{37}$

Similar to studies conducted on other anticoagulants, the otolaryngology literature focuses on flap survival and hematoma rather than DVT prevention. ${ }^{38}$ Motakef et $\mathrm{al}^{38}$ concluded that the use of HSQ is an efficacious option for thromboprophylaxis in microsurgery, and a range of doses have been proven safe $(5,000 \mathrm{U}$ once, twice, or three times daily), although there is no true consensus on dose, duration, or initiation of therapy. ${ }^{33,39}$ Stockmans et al demonstrated that higher doses of HSQ can be more effective in preventing VTE but may come with an increased incidence of bleeding. ${ }^{37}$

Most of the existing literature demonstrate that HSQ is not associated with an increase in adverse flap outcomes. ${ }^{30,31,40,41}$ The landmark multicenter prospective study on head and neck microsurgery by Khouri et al, one of the only level-I evidence studies addressing anticoagulation protocols, demonstrated that only HSQ had a significant protective effect on flap thrombosis. ${ }^{25}$ A recent review by Fan et al concluded that there are insufficient data to refute this evidence. ${ }^{42}$ A recent meta-analysis evaluating the efficacy of thromboprophylactic agents used in microsurgery concluded that HSQ reduced the risk of flap loss by $35 \%$ but raised the risk of hematoma, though these differences were significant. ${ }^{30}$ In a retrospective review of 470 patients undergoing free tissue transfer, HSQ was found to be a safe agent that did not increase the risk of postoperative bleeding, hematoma, or flap failure. ${ }^{31}$ A meta-analysis conducted by Pan et al analyzing large-scale retrospective studies of anticoagulation regimens in microvascular reconstruction demonstrated that the flap loss rate is similar when heparin or aspirin is used. ${ }^{41} \mathrm{~A}$ review of the free flap literature from 1996 to 2005 recommended that anticoagulation, preferably with heparin, was a required part of postoperative management. ${ }^{40}$

Recently, a Cochrane review was conducted comparing LMWH to HSQ for perioperative thromboprophylaxis for patients with cancer and found no significant difference in outcomes between the agents. ${ }^{43}$ In their retrospective cohort study, Bahl et $\mathrm{al}^{44}$ showed that patients undergoing free tissue transfer reduced their incidence of DVT from 7.7 to $2.1 \%$ but increased their bleeding complications from 4.5 to $11.9 \%$ when receiving chemoprophylaxis consisting of either HSQ or LMWH. Importantly, increased bleeding was associated with concomitant usage of antiplatelet medications and anticoagulants. This study, however, did not evaluate the difference in effect between HSQ and LMWH, and patients were also treated with various antiplatelet and perioperative chemoprophylactic agents, thus making results difficult to interpret. $^{44}$

HSQ is an efficacious option for perioperative DVT prophylaxis that is widely used, and no prospective or large-scale 
studies have demonstrated a detrimental effect of heparin compared with other agents regarding flap survival.

\section{Intravenous Heparin}

Intravenous (IV) heparin is the primary treatment for active VTE. No trials or reviews exist demonstrating the use of a heparin infusion for VTE prevention in head and neck surgery. However, its role in flap thromboprophylaxis is better described. Several studies have shown that patients treated with heparin drips following free tissue transfer surgery had significantly increased complications, including flap loss and hematoma/hemorrhage, compared with those who did not receive prophylaxis. ${ }^{15}$ Kroll et al described the use of a continuous heparin infusion and concluded that doses above $500 \mathrm{U} /$ hour increase significantly the risk of hematoma. ${ }^{45}$ Nelson et al compared the use of an intraoperative heparin bolus and postoperative heparin infusion to HSQ in 23 hypercoagulable patients undergoing microsurgery. The cohort treated with IV heparin had clinically lower rates of VTE and flap loss but an increased risk for postoperative bleeding, hematoma, and anemia. ${ }^{46}$

\section{Low-Molecular-Weight Heparins}

LMWH is a fragmented heparin derivative with a more specific inhibitory effect on factor $\mathrm{X}$ and a less systemic effect on antithrombin activity.

Within the DVT literature, there have been multiple studies demonstrating LMWH to be at least as effective as UFH for thromboprophylaxis. ${ }^{43}$ This, along with a favorable risk profile initially demonstrated by Malm et $\mathrm{al}^{47}$ in an animal model study on surgical bleeding rates, has made LMWH a practicechanging treatment strategy. LMWH has a more predictable dose-response relationship, greater bioavailability, and longer-half life and is thus preferable for high-risk patients. ${ }^{48,49}$ The ACCP recommends either LMWH or low-dose UFH for high-risk patients undergoing general surgery and LMWH only for patients undergoing high-risk orthopaedic surgery. ${ }^{11}$ Increased dosing of LMWH in patients who are at high risk, such as those with malignancy or undergoing microvascular reconstruction, has also been recommended. ${ }^{50}$

Currently, factor Xa levels are being used to modulate therapy. Eley et $\mathrm{al}^{51}$ concluded that there was a high incidence of subtherapeutic anti-Xa levels in response to standard prophylactic doses of dalteparin in 153 head and neck microvascular patients but showed success in dose adjustment of LMWH by using anti-Xa concentrations to titrate a standard 2,500 U dose to therapeutic concentrations. However, the accuracy of such measurements may be variable, as levels must be drawn exactly after LMWH is administered. ${ }^{51}$ There also exist multiple formulations of LMWH in the market, making standardization more difficult. ${ }^{52,53}$

Similar to other agents, there are no studies designed specifically to evaluate DVT outcomes in free tissue transfer patients treated with LMWH. The orthopaedic literature cites several trials demonstrating that LMWH reduces the incidence of DVT in hip replacement surgery by 31 to $79 \%$ compared with placebo and is as effective or more effective than $\mathrm{HSQ}^{54,55}$ One randomized controlled trial of 665 patients undergoing hip replacement showed fewer bleeding events in patients given LMWH (30 mg twice daily) compared with HSQ (7,500 U twice daily) and no difference compared with placebo. ${ }^{56}$ In a randomized prospective study of critical patients undergoing major surgery, LMWH (40 mg once daily) and HSQ (5,000 U twice daily) were found to be equivalent as prophylaxis against DVT; UFH did result in a greater risk of hematoma. ${ }^{49} \mathrm{~A}$ meta-analysis of general surgery patients treated with LMWH showed a lesser incidence of PE but a trend toward an increase in bleeding complications. ${ }^{48}$ In a study of 225 patients following breast microvascular reconstruction, Lemaine et al showed a $3.4 \%$ incidence of clinically silent DVT and a $1.9 \%$ rate of flap loss with $\mathrm{LMWH}^{57}$

Multiple studies have addressed the effect of LMWH on flap outcomes. In a study of 30 head and neck microvascular patients, Karimi et al demonstrated the utility of a combination protocol of aspirin and enoxaparin for 5 and 3 days, respectively, for flap survival, with no incidence of flap loss or hematoma. ${ }^{58} \mathrm{~A}$ retrospective study of 137 microvascular reconstruction patients demonstrated that $\mathrm{LMWH}$ is an effective standalone antithrombotic, with outcomes comparable to those of HSQ and aspirin studies and a flap survival rate of $97.1 \% .^{59}$ In a study of 153 microvascular head and neck patients receiving prophylactic doses of dalteparin with or without aspirin, there were six events of hematoma, and this risk was not influenced by the dose of dalteparin or addition of aspirin. ${ }^{51}$ In a systematic review by Pršić et al, ${ }^{60}$ attention is drawn to the conflicting results of studies on LMWH for flap thrombosis, with some demonstrating equal if not better outcomes than HSQ such as decreased rate of bleeding, and others showing it to be significantly inferior.

To date, there is an ongoing debate in the literature regarding $\mathrm{LMWH}$, but an improved risk profile and the potential of decreased bleeding compared with UFH make this agent a viable thromboprophylactic option in head and neck microvascular surgery, particularly for patients in whom HSQ is contraindicated or who possess high-risk factors requiring a more aggressive thromboprophylactic regimen.

\section{Dextran}

While no data have been reported on the use of dextran for systemic DVT, multiple studies have focused on microvascular thromboprophylaxis.

Dextran, by impairing platelet function, prolonging bleeding time, and destabilizing fibrin polymerization, has been shown to reduce the incidence of microanastomotic thrombosis in multiple animal models. ${ }^{61-63}$ However, results from clinical studies have largely suggested that dextran has no bearing on the success rates of free tissue transfer in head and neck reconstruction ${ }^{64-68}$ and may even increase flap loss. ${ }^{45} \mathrm{~A}$ recent large study by Riva et al, ${ }^{67}$ comparing three groups of patients in which prostaglandin-E1, dextran 40 , and no prophylaxis were given, concluded that neither PGE1 nor dextran benefits flap survival. In a prospective randomized trial, Disa et $\mathrm{al}^{64}$ determined that there was no difference in overall flap survival between dextran and aspirin use.

Serious complications of dextran have been reported in the literature, such as bleeding diatheses in children, osmotic 
complications, pulmonary edema, cerebral edema, nephrotoxicity, and fatal anaphylaxis. ${ }^{69-73}$ Disa et $\mathrm{al}^{64}$ concluded that patients receiving dextran had up to a 7.2 greater relative risk of developing an adverse cardiac or pulmonary event, especially acute respiratory distress syndrome, when compared with patients receiving aspirin. The frequency of systemic complications was also shown to correlate positively with the duration of dextran use. Jayaprasad et $\mathrm{al}^{65}$ reported a statistically significant increase in postoperative atelectasis but no other local or systemic complications. Fan et al identified level-I evidence showing dextran to increase systemic complications. ${ }^{42}$ Despite this, a questionnaire conducted in 2007 among head and neck surgeons in the United Kingdom demonstrated that $45 \%$ of respondents use dextran routinely and nearly $20 \%$ combine it with other antithrombotic agents such as aspirin and heparin. ${ }^{66}$

Studies assessing the efficacy of dextran as DVT prophylaxis in orthopaedic procedures can also inform its potential utility in head and neck microvascular reconstructive surgery. In their systematic review evaluating various methods of thromboprophylaxis, Roderick et al ${ }^{16}$ ascertained that dextran decreases the risk of DVT by approximately 50\% regardless of the type of surgery, but no reliable conclusions could be drawn regarding PE. Dextran appeared to be less effective in preventing DVT than the heparin regimens employed in the included studies. ${ }^{74-76}$ Dextran was also associated with an increased risk of bleeding. One study conducted in geriatric hip fracture patients showed that aspirin and dextran are equally effective thromboembolic prophylactic agents. ${ }^{77}$

\section{Warfarin}

The use of warfarin as DVT prophylaxis is not described in any study. It is a mainstay of treatment only once DVT is established.

\section{Inferior Vena Cava Filter}

No literature exists on the use of inferior vena cava (IVC) filters for VTE prophylaxis in head and neck surgery. IVC filters are indicated in patients with proven thromboembolic disease and with contraindications to anticoagulation, complications of anticoagulation, or recurrent VTE. ${ }^{78}$

\section{Discussion}

A majority of otolaryngology patients undergoing microvascular reconstruction possess all three cardinal factors of Virchow's triad-venous stasis secondary to prolonged operations especially when involving free tissue transfer, hypercoagulability as a result of malignancy, and endothelial damage from tumor resection and tissue harvesting. ${ }^{79}$ The exact occurrence of DVT in these patients has, however, been debated and inadequately addressed. A retrospective study of 1,018 patients who underwent HNC surgery reported no VTE events and 12 bleeding complications, 11 of which occurred in patients who received chemoprophylaxis, and concluded that chemoprophylaxis should not be routinely used. ${ }^{80}$ A recent systematic review of 23 articles encompassing 618,264 otolaryngology surgery patients showed a VTE incidence of $0.4 \%$ and concluded that chemoprophylaxis did not decrease the risk of VTE but increased the risk of bleeding. ${ }^{81}$ On the other hand, the greatest incidence of DVT has been shown to occur in patients undergoing comprehensive resection with reconstruction., ${ }^{3,5,7}$ One study demonstrated a fourfold increase in DVT in patients undergoing head and neck reconstruction. ${ }^{4}$

The role of chemoprophylaxis with anticoagulants in patients undergoing microvascular reconstruction is particularly challenging, given the potential failure of microvascular reconstruction from bleeding or hematoma. Other possible adverse sequelae include airway compromise and wound complications. ${ }^{10}$ These risks, along with the perception of low DVT incidence in this population, have been identified as factors contributing to the controversy of routine chemoprophylaxis following microvascular reconstruction of the head and neck. Ah-See et $\mathrm{al}^{82}$ reported in 1997 that a majority of otolaryngologists do not employ any form of DVT prophylaxis, although more recent studies have shown an increase in the practice.

VTE thromboprophylaxis protocols in head and neck microvascular reconstruction have received sparse attention in the otolaryngology literature. Many studies ${ }^{13}$ focus only on the effects of chemoprophylaxis on outcomes of free tissue transfer, and there are no long-term studies addressing the incidence of DVT. However, a recent systematic review and meta-analysis opined that no postoperative antithrombotic regimen has shown significant benefits for flap survival. ${ }^{30}$ Therefore, there is a need to shift focus to VTE prevention. If an effective VTE prophylactic regimen demonstrates typical flap survival, it should be adopted as a standard of care.

Microvascular surgeons have experimented with various regimens, many of which are a combination of the methods used in previous randomized controlled trials in other patient populations and expert opinion. The armamentarium encompasses IPC, early ambulation, aspirin, HSQ LMWH, systemic anticoagulation, dextran, and IVC filters. Agents used in chemoprophylaxis target the coagulation cascade primarily (-Fig. 1). The coagulation cascade has two pathways, intrinsic and extrinsic, that are a series of reactions in which an inactive serine protease and its glycoprotein cofactor are activated and subsequently catalyze the next reaction in the cascade, ultimately leading to cross-linked fibrin. ${ }^{83}$

The importance of developing a reliable tool for stratifying DVT risk cannot be overemphasized, as it can guide subsequent aggressiveness of postoperative thromboprophylaxis. One method for stratifying the risk of DVT that is often described in the literature is the Caprini risk assessment model, ${ }^{9,44,84}$ which uses a point scoring system for approximately 40 risk factors. The Caprini risk assessment tool has been validated in both retrospective and prospective studies for patients undergoing elective general surgery and modified for use in plastic surgery patients. ${ }^{44,85}$ Studies have consistently shown that patients with a high Caprini risk score have a higher rate of DVT. 5, 7,44,84 Two studies have also demonstrated its effectiveness for identifying patients with low, moderate, and high risk for DVT within 30 days after surgery. ${ }^{44,84}$ However, results often did not reach statistical significance. ${ }^{5,9,44}$ 


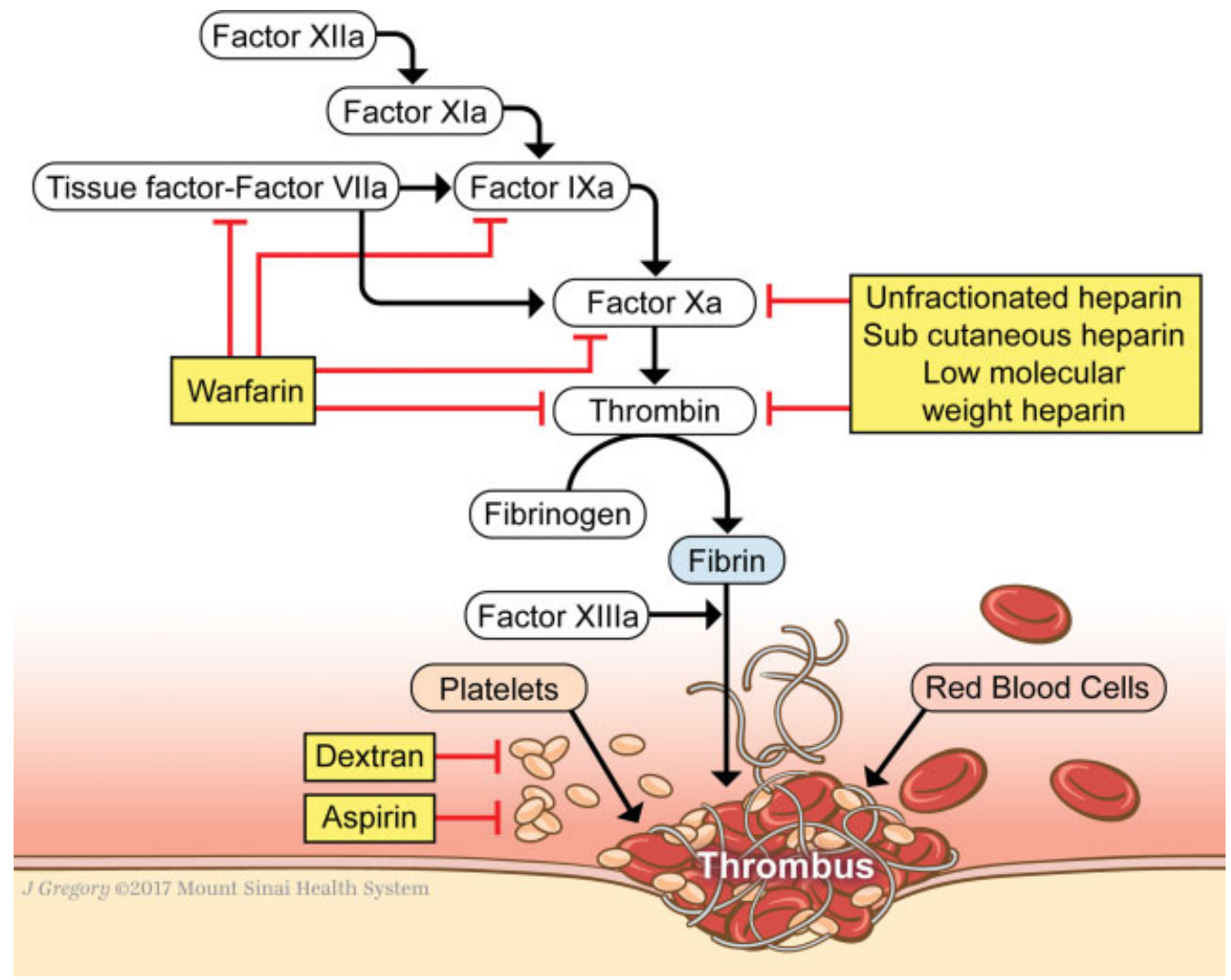

Fig. 1 Schematic of the coagulation cascade and target areas of chemoprophylactic agents. (We thank Jill Gregory for providing this artwork. This piece has been used with permission from the Mount Sinai Health System).

There are caveats to the routine use of early ambulation and mechanical methods such as IPC. Specifically, patients undergoing fibula tissue transfer are likely to be immobilized for a period of time following surgery. In addition, IPC is contraindicated on the donor limb. Other adjunct therapies may thus be warranted in these patients who are at higher risk for DVT. When anticoagulation is precluded, such as in patients with bleeding diathesis, hemorrhagic stroke, or complications of anticoagulation, an IVC filter may play an important role. 86

There is a lack of substantial evidence to show that drugs used to primarily treat local thrombosis (aspirin, dextran) are also able to reduce the risk of DVT/PE as effectively as other thromboprophylactic medications (HSQ LMWH), especially when utilized in single pharmacotherapy regimens. However, several studies have concluded that a combination of aspirin and a systemic anticoagulant, while effective in DVT prevention, may increase the rate of hematoma. Thus, the combined use of aspirin and an anticoagulant must be approached cautiously. Analysis of the current studies that exist in the microvascular, head and neck, and otolaryngology literature shows most evidence for the use of HSQ and mechanical prophylaxis, with at least equivalent flap survival rates compared with no chemoprophylaxis.

Meta-analyses comparing UFH and LMWH have shown both agents to be effective as primary VTE prophylaxis. ${ }^{87}$ Both the ACCP and the American Society of Clinical Oncology endorse routine chemoprophylaxis with LMWH in cancer patients undergoing surgery, who are at increased risk for VTE. $^{88}$ Studies are currently focusing on the superiority of LMWH over UFH for general thromboprophylaxis. ${ }^{89}$ In addition, new clinical studies have proposed continuation of LMWH thromboprophylaxis for up to 3 weeks after discharge, which may reduce the rate of late DVT by $60 \%{ }^{87}$ There may also be a unique role for LMWH in patients with no risk factors for bleeding, a history of heparin-induced thrombocytopenia, or active VTE. However, more studies specific to head and neck microvascular reconstruction need to be conducted before official recommendations are made regarding LMWH.

Novel agents are being presented regularly to improve both flap survival and patient outcomes in microvascular surgery. Pršić et al supported the use of statins as part of the perioperative regimen for patients undergoing microvascular reconstruction. ${ }^{60}$ Wikner et al studied the role of thromboelastometry in microsurgery. ${ }^{90}$ Thromboelastometry is a viscoelastic hemostatic assay that provides a graphical evaluation of the kinetics of clot formation, ideally allowing distinction between a surgical etiology of bleeding and coagulopathy, diagnosis of the type of coagulopathy, and guidance in treatment. ${ }^{91-93}$ Both of these examples suggest how perioperative free flap management protocols may evolve in the future.

The ideal study of randomizing patients to different anticoagulation regimens and analyzing VTE and free tissue transfer survival outcomes has yet to be performed. Thus, this assessment is based on the most meaningful conclusions drawn from 
studies that do exist. The regimens with the most consistent support in the literature are HSQ mechanical methods, and early mobilization. Aspirin and dextran have not been shown to aid in microvascular flap survival, and their role in VTE prophylaxis is equivocal; a stratified, patient-specific risk-benefit analysis should thus be considered. LMWH has demonstrated effectiveness in specific patient populations and its use as chemoprophylaxis in patients undergoing microvascular reconstructive surgery is increasing. Ultimately, more controlled studies comparing both individual agents and no prophylaxis must be conducted.

\section{Conclusion}

There is a clear need for a consistent chemoprophylaxis protocol for patients undergoing microvascular reconstruction. While many agree therapy should be tailored to the individual patient, the establishment of consensus guidelines similar to those recommended by the ACCP among those specializing in the field would improve patient management. As microvascular reconstruction is becoming a more successful and common procedure, evidence must now be applied to the art.

Most data in the literature support the routine use of HSQ and mechanical methods as VTE prophylaxis. HSQ and aspirin are commonly used in tandem, but there is evidence that combining antithrombotic agents increases the rate of complications and aspirin alone does not increase flap survival. There have been recent studies demonstrating the efficacy, safety, and decreased risk of bleeding with LMWH, which may have a role in more vulnerable populations, but the literature is insufficient to recommend its use across all head and neck microvascular reconstructive surgery. Multiinstitutional, randomized clinical trials with controls must be conducted to corroborate the conclusions presented in this review article.

\section{Conflict of Interest}

This article has not been/nor is being considered for publication elsewhere. There are no financial disclosures or conflicts of interest.

\section{Acknowledgment}

We thank Jill Gregory, Senior Medical Illustrator, from the Mount Sinai Health System for providing artwork for this project.

\section{References}

1 Agnelli G. Prevention of venous thromboembolism in surgical patients. Circulation 2004;110(24, Suppl 1):IV4-IV12

2 Geerts WH, Pineo GF, Heit JA, et al. Prevention of venous thromboembolism: the Seventh ACCP Conference on Antithrombotic and Thrombolytic Therapy. Chest 2004;126(3, Suppl):338S-400S

3 Ali NS, Nawaz A, Junaid M, Kazi M, Akhtar S. Venous thromboembolism-incidence of deep venous thrombosis and pulmonary embolism in patients with head and neck cancer: a tertiary care experience in Pakistan. Int Arch Otorhinolaryngol 2015; 19(03):200-204
4 Chen CM, Disa JJ, Cordeiro PG, Pusic AL, McCarthy CM, Mehrara BJ. The incidence of venous thromboembolism after oncologic head and neck reconstruction. Ann Plast Surg 2008;60(05):476-479

5 Clayburgh DR, Stott W, Cordiero T, et al. Prospective study of venous thromboembolism in patients with head and neck cancer after surgery. JAMA Otolaryngol Head Neck Surg 2013;139(11): $1143-1150$

6 Garritano FG, Lehman EB, Andrews GA. Incidence of venous thromboembolism in otolaryngology-head and neck surgery. JAMA Otolaryngol Head Neck Surg 2013;139(01):21-27

7 Kakei Y, Akashi M, Hasegawa T, Minamikawa T, Usami S, Komori T. Incidence of venous thromboembolism after oral oncologic surgery with simultaneous reconstruction. J Oral Maxillofac Surg 2016;74(01):212-217

8 Moreano EH, Hutchison JL, McCulloch TM, Graham SM, Funk GF, Hoffman HT. Incidence of deep venous thrombosis and pulmonary embolism in otolaryngology-head and neck surgery. Otolaryngol Head Neck Surg 1998;118(06):777-784

9 Thai L, McCarn K, Stott W, et al. Venous thromboembolism in patients with head and neck cancer after surgery. Head Neck 2013;35(01):4-9

10 Pugh CM, Dennis RH II, Massac EA. Evaluation of intraoperative anticoagulants in microvascular free-flap surgery. J Natl Med Assoc 1996;88(10):655-657

11 Gould MK, Garcia DA, Wren SM, et al. Prevention of VTE in nonorthopedic surgical patients: Antithrombotic Therapy and Prevention of Thrombosis, 9th ed: American College of Chest Physicians Evidence-Based Clinical Practice Guidelines. Chest 2012;141(2, Suppl):e227S-e277S

12 Garritano FG, Andrews GA. Current practices in venous thromboembolism prophylaxis in otolaryngology-head and neck surgery. Head Neck 2016;38(Suppl 1):E341-E345

13 Innis WP, Anderson TD. Deep venous thrombosis and pulmonary embolism in otolaryngologic patients. Am J Otolaryngol 2009;30 (04):230-233

14 Lee J, Alexander A, Higgins K, Geerts W. The Sunnybrook experience: review of deep vein thrombosis and pulmonary embolism in otolaryngology.J Otolaryngol Head Neck Surg 2008;37(04):547-551

15 Lighthall JG, Cain R, Ghanem TA, Wax MK. Effect of postoperative aspirin on outcomes in microvascular free tissue transfer surgery. Otolaryngol Head Neck Surg 2013;148(01):40-46

16 Roderick P, Ferris G, Wilson K, et al. Towards evidence-based guidelines for the prevention of venous thromboembolism: systematic reviews of mechanical methods, oral anticoagulation, dextran and regional anaesthesia as thromboprophylaxis. Health Technol Assess 2005;9(49):iii-iv, ix-x, 1-78

17 Clagett GP, Anderson FA Jr, Levine MN, Salzman EW, Wheeler HB. Prevention of venous thromboembolism. Chest 1992;102 (4, Suppl):391S-407S

18 Kruse AL, Luebbers HT, Grätz KW, Obwegeser JA. Factors influencing survival of free-flap in reconstruction for cancer of the head and neck: a literature review. Microsurgery 2010;30(03):242-248

19 Yeung JK, Harrop R, McCreary O, et al. Delayed mobilization after microsurgical reconstruction: an independent risk factor for pneumonia. Laryngoscope 2013;123(12):2996-3000

20 Undas A, Brummel-Ziedins KE, Mann KG. Antithrombotic properties of aspirin and resistance to aspirin: beyond strictly antiplatelet actions. Blood 2007;109(06):2285-2292

21 Wakefield TW, Obi AT, Henke PK. An aspirin a day to keep the clots away: can aspirin prevent recurrent thrombosis in extended treatment for venous thromboembolism? Circulation 2014;130 (13):1031-1033

22 Becattini C, Agnelli G, Schenone A, et al; WARFASA Investigators. Aspirin for preventing the recurrence of venous thromboembolism. N Engl J Med 2012;366(21):1959-1967

23 Brighton TA, Eikelboom JW, Mann K, et al; ASPIRE Investigators. Low-dose aspirin for preventing recurrent venous thromboembolism. N Engl J Med 2012;367(21):1979-1987 
24 Johnson PC, Barker JH. Thrombosis and antithrombotic therapy in microvascular surgery. Clin Plast Surg 1992;19(04):799-807

25 Khouri RK, Cooley BC, Kunselman AR, et al. A prospective study of microvascular free-flap surgery and outcome. Plast Reconstr Surg 1998;102(03):711-721

26 Li X, Cooley BC. Effect of anticoagulation and inhibition of platelet aggregation on arterial versus venous microvascular thrombosis. Ann Plast Surg 1995;35(02):165-169, discussion 169-170

27 Spiegel JH, Polat JK. Microvascular flap reconstruction by otolaryngologists: prevalence, postoperative care, and monitoring techniques. Laryngoscope 2007;117(03):485-490

28 Blackwell KE. Unsurpassed reliability of free flaps for head and neck reconstruction. Arch Otolaryngol Head Neck Surg 1999;125 (03):295-299

29 Bahl V, Shuman AG, Hu HM, et al. Chemoprophylaxis for venous thromboembolism in otolaryngology. JAMA Otolaryngol Head Neck Surg 2014;140(11):999-1005

30 Lee KT, Mun GH. The efficacy of postoperative antithrombotics in free flap surgery: a systematic review and meta-analysis. Plast Reconstr Surg 2015;135(04):1124-1139

31 Ashjian P, Chen CM, Pusic A, Disa JJ, Cordeiro PG, Mehrara BJ. The effect of postoperative anticoagulation on microvascular thrombosis. Ann Plast Surg 2007;59(01):36-39, discussion 39-40

32 Dort JC, Farwell DG, Findlay M, et al. Optimal perioperative care in major head and neck cancer surgery with free flap reconstruction: a consensus review and recommendations from the enhanced recovery after surgery society. JAMA Otolaryngol Head Neck Surg 2017;143(03):292-303

33 Chien W, Varvares MA, Hadlock T, Cheney M, Deschler DG. Effects of aspirin and low-dose heparin in head and neck reconstruction using microvascular free flaps. Laryngoscope 2005;115(06): 973-976

34 Veravuthipakorn L, Veravuthipakorn A. Microsurgical free flap and replantation without antithrombotic agents. J Med Assoc Thai 2004;87(06):665-669

35 Kumar S, Moorthy R. New oral anticoagulants - a guide for ENT surgeons. J Laryngol Otol 2016;130(04):324-328

36 Askari M, Fisher C, Weniger FG, Bidic S, Lee WP. Anticoagulation therapy in microsurgery: a review. J Hand Surg Am 2006;31(05): 836-846

37 Stockmans F, Stassen JM, Vermylen J, Hoylaerts MF, Nyström A. A technique to investigate microvascular mural thrombus formation in arteries and veins: II. Effects of aspirin, heparin, r-hirudin, and G-4120. Ann Plast Surg 1997;38(01):63-68

38 Motakef S, Mountziaris PM, Ismail IK, Agag RL, Patel A. Emerging paradigms in perioperative management for microsurgical free tissue transfer: review of the literature and evidence-based guidelines. Plast Reconstr Surg 2015;135(01):290-299

39 Xipoleas G, Levine E, Silver L, Koch RM, Taub PJ. A survey of microvascular protocols for lower-extremity free tissue transfer I: perioperative anticoagulation. Ann Plast Surg 2007;59(03): 311-315

40 Lecoq JP, Senard M, Hartstein GM, Lamy M, Heymans O. Thromboprophylaxis in microsurgery. Acta Chir Belg 2006;106(02): 158-164

41 Pan XL, Chen GX, Shao HW, Han CM, Zhang LP, Zhi LZ. Effect of heparin on prevention of flap loss in microsurgical free flap transfer: a meta-analysis. PLoS One 2014;9(04):e95111

42 Fan KL, Patel KM, Mardini S, Attinger C, Levin LS, Evans KK. Evidence to support controversy in microsurgery. Plast Reconstr Surg 2015;135(03):595e-608e

43 Akl EA, Kahale L, Sperati F, et al. Low molecular weight heparin versus unfractionated heparin for perioperative thromboprophylaxis in patients with cancer. Cochrane Database Syst Rev 2014; (06):CD009447

44 Bahl V, Hu HM, Henke PK, Wakefield TW, Campbell DA Jr, Caprini JA. A validation study of a retrospective venous thromboembolism risk scoring method. Ann Surg 2010;251(02):344-350
45 Kroll SS, Miller MJ, Reece GP, et al. Anticoagulants and hematomas in free flap surgery. Plast Reconstr Surg 1995;96(03):643-647

46 Nelson JA, Chung CU, Bauder AR, Wu LC. Prevention of thrombosis in hypercoagulable patients undergoing microsurgery: a novel anticoagulation protocol. J Plast Reconstr Aesthet Surg 2017;70 (03):307-312

47 Malm K, Dahlbäck B, Arnljots B. Low-molecular-weight heparin (dalteparin) effectively prevents thrombosis in a rat model of deep arterial injury. Plast Reconstr Surg 2003;111(05): 1659-1666

48 Green D, Hirsh J, Heit J, Prins M, Davidson B, Lensing AW. Low molecular weight heparin: a critical analysis of clinical trials. Pharmacol Rev 1994;46(01):89-109

49 De A, Roy P, Garg VK, Pandey NK. Low-molecular-weight heparin and unfractionated heparin in prophylaxis against deep vein thrombosis in critically ill patients undergoing major surgery. Blood Coagul Fibrinolysis 2010;21(01):57-61

50 Blackburn TK, Java KR, Lowe D, Brown JS, Rogers SN. Safety of a regimen for thromboprophylaxis in head and neck cancer microvascular reconstructive surgery: non-concurrent cohort study. $\mathrm{Br}$ J Oral Maxillofac Surg 2012;50(03):227-232

51 Eley KA, Parker RJ, Watt-Smith SR. Low molecular weight heparin in patients undergoing free tissue transfer following head and neck ablative surgery: review of efficacy and associated complications. Br J Oral Maxillofac Surg 2013;51(07):610-614

52 Mulloy B, Hogwood J, Gray E, Lever R, Page CP. Pharmacology of heparin and related drugs. Pharmacol Rev 2016;68(01): 76-141

53 Nader HB, Walenga JM, Berkowitz SD, Ofosu F, Hoppensteadt DA Cella G. Preclinical differentiation of low molecular weight heparins. Semin Thromb Hemost 1999;25(Suppl 3):63-72

54 Planes A, Vochelle N, Darmon JY. Out-of-hospital prophylaxis with low-molecular-weight heparin in hip surgery: the French studyvenographic outcome at 35 days. Chest 1998;114(2, Suppl Evidence):125S-129S

55 Spiro TE, Johnson GJ, Christie MJ, et al; Enoxaparin Clinical Trial Group. Efficacy and safety of enoxaparin to prevent deep venous thrombosis after hip replacement surgery. Ann Intern Med 1994; 121(02):81-89

56 Levine MN, Hirsh J, Gent M, et al. Prevention of deep vein thrombosis after elective hip surgery. A randomized trial comparing low molecular weight heparin with standard unfractionated heparin. Ann Intern Med 1991;114(07):545-551

57 Lemaine V, McCarthy C, Kaplan K, et al. Venous thromboembolism following microsurgical breast reconstruction: an objective analysis in 225 consecutive patients using low-molecular-weight heparin prophylaxis. Plast Reconstr Surg 2011;127(04): 1399-1406

58 Karimi E, Ardestani SH, Jafari M, Hagh AB. Testing a new anticoagulation method for free flap reconstruction of head and neck cancers. Clin Exp Otorhinolaryngol 2016;9(04):370-373

59 Reiter M, Kapsreiter M, Betz CS, Harréus U. Perioperative management of antithrombotic medication in head and neck reconstruction-a retrospective analysis of 137 patients. Am J Otolaryngol 2012;33(06):693-696

60 Pršić A, Kiwanuka E, Caterson SA, Caterson EJ. Anticoagulants and statins as pharmacological agents in free flap surgery: current rationale. Eplasty 2015;15:e51

61 Salemark L, Wieslander JB, Dougan P, Arnljots B. Studies of the antithrombotic effects of dextran 40 following microarterial trauma. Br J Plast Surg 1991;44(01):15-22

62 Scholz T, Evans GR. Impact of hypertonic and hyperoncotic saline solutions on ischemia-reperfusion injury in free flaps. Plast Reconstr Surg 2008;122(01):85-94

63 Wieslander JB, Dougan P, Stjernquist U, Aberg M, Bergentz SE. The influence of dextran and saline solution upon platelet behavior after microarterial anastomosis. Surg Gynecol Obstet 1986; 163(03):256-262 
64 Disa JJ, Polvora VP, Pusic AL, Singh B, Cordeiro PG. Dextran-related complications in head and neck microsurgery: do the benefits outweigh the risks? A prospective randomized analysis. Plast Reconstr Surg 2003;112(06):1534-1539

65 Jayaprasad K, Mathew J, Thankappan K, et al. Safety and efficacy of low molecular weight dextran (dextran 40) in head and neck free flap reconstruction. J Reconstr Microsurg 2013;29(07):443-448

66 Ridha H, Jallali N, Butler PE. The use of dextran post free tissue transfer. J Plast Reconstr Aesthet Surg 2006;59(09):951-954

67 Riva FM, Chen YC, Tan NC, et al. The outcome of prostaglandin-E1 and dextran-40 compared to no antithrombotic therapy in head and neck free tissue transfer: analysis of 1,351 cases in a single center. Microsurgery 2012;32(05):339-343

68 Sun TB, Chien SH, Lee JT, Cheng LF, Hsu LP, Chen PR. Is dextran infusion as an antithrombotic agent necessary in microvascular reconstruction of the upper aerodigestive tract? J Reconstr Microsurg 2003;19(07):463-466

69 Hardin CK, Kirk WC, Pederson WC. Osmotic complications of lowmolecular-weight dextran therapy in free flap surgery. Microsurgery 1992;13(01):36-38

70 Hein KD, Wechsler ME, Schwartzstein RM, Morris DJ. The adult respiratory distress syndrome after dextran infusion as an antithrombotic agent in free TRAM flap breast reconstruction. Plast Reconstr Surg 1999;103(06):1706-1708

71 Kaplan AI, Sabin S. Dextran 40: another cause of drug-induced noncardiogenic pulmonary edema. Chest 1975;68(03):376-377

72 Machado MA, Volpe P, Lima M das G, et al. [Anaphylaxis after dextran 40 infusion: report of a case and review of the literature]. Rev Hosp Clin Fac Med Sao Paulo 1993;48(04):167-169

73 Parry SW, Toth BA, Elliott LF. Microvascular free-tissue transfer in children. Plast Reconstr Surg 1988;81(06):838-840

74 Francis CW, Pellegrini VD Jr, Stulberg BN, Miller ML, Totterman S, Marder VJ. Prevention of venous thrombosis after total knee arthroplasty. Comparison of antithrombin III and low-dose heparin with dextran. J Bone Joint Surg Am 1990;72(07):976-982

75 Mätzsch T, Bergqvist D, Fredin H, Hedner U. Low molecular weight heparin compared with dextran as prophylaxis against thrombosis after total hip replacement. Acta Chir Scand 1990;156 (6-7):445-450

76 Oertli D, Hess P, Durig M, et al. Prevention of deep vein thrombosis in patients with hip fractures: low molecular weight heparin versus dextran. World J Surg 1992;16(05):980-984, discussion 984-985

77 Feldman DS, Zuckerman JD, Walters I, Sakales SR. Clinical efficacy of aspirin and dextran for thromboprophylaxis in geriatric hip fracture patients. J Orthop Trauma 1993;7(01):1-5

78 Büller HR, Agnelli G, Hull RD, Hyers TM, Prins MH, Raskob GE. Antithrombotic therapy for venous thromboembolic disease: the Seventh ACCP Conference on Antithrombotic and Thrombolytic Therapy. Chest 2004;126(3, Suppl):401S-428S
79 Jaggi R, Taylor SM, Trites J, Anderson D, MacDougall P, Hart RD. Review of thromboprophylaxis in otolaryngology-head and neck surgery. J Otolaryngol Head Neck Surg 2011;40(03):261-265

80 Gavriel H, Thompson E, Kleid S, Chan S, Sizeland A. Safety of thromboprophylaxis after oncologic head and neck surgery. Study of 1018 patients. Head Neck 2013;35(10):1410-1414

81 Moubayed SP, Eskander A, Mourad MW, Most SP. Systematic review and meta-analysis of venous thromboembolism in otolaryngology-head and neck surgery. Head Neck 2017;39(06): $1249-1258$

82 Ah-See KW, Kerr J, Sim DW. Prophylaxis for venous thromboembolism in head and neck surgery: the practice of otolaryngologists. J Laryngol Otol 1997;111(09):845-849

83 Pallister C, Watson M. Haematology. 2nd ed: Scion Publishing; 2010

84 Shuman AG, Hu HM, Pannucci CJ, Jackson CR, Bradford CR, Bahl V. Stratifying the risk of venous thromboembolism in otolaryngology. Otolaryngol Head Neck Surg 2012;146(05):719-724

85 Davison SP, Venturi ML, Attinger CE, Baker SB, Spear SL. Prevention of venous thromboembolism in the plastic surgery patient. Plast Reconstr Surg 2004;114(03):43E-51E

86 Young T, Aukes J, Hughes R, Tang H. Vena caval filters for the prevention of pulmonary embolism. Cochrane Database Syst Rev 2007;(03):CD006212

87 Robert F. The potential benefits of low-molecular-weight heparins in cancer patients. J Hematol Oncol 2010;3(01):3

88 Lee AYY, Levine MN, Baker RI, et al; Randomized Comparison of Low-Molecular-Weight Heparin versus Oral Anticoagulant Therapy for the Prevention of Recurrent Venous Thromboembolism in Patients with Cancer (CLOT) Investigators. Low-molecular-weight heparin versus a coumarin for the prevention of recurrent venous thromboembolism in patients with cancer. N Engl J Med 2003;349 (02):146-153

89 Akl EA, Terrenato I, Barba M, et al. Low-molecular-weight heparin vs unfractionated heparin for perioperative thromboprophylaxis in patients with cancer: a systematic review and meta-analysis. Arch Intern Med 2008;168(12):1261-1269

90 Wikner J, Beck-Broichsitter BE, Schlesinger S, et al. Thromboelastometry: a contribution to perioperative free-flap management. J Craniomaxillofac Surg 2015;43(07):1065-1071

91 Benes J, Zatloukal J, Kletecka J. Viscoelastic methods of blood clotting assessment - a multidisciplinary review. Front Med (Lausanne) 2015;2:62

92 Luddington RJ. Thrombelastography/thromboelastometry. Clin Lab Haematol 2005;27(02):81-90

93 Wikkelsø A, Wetterslev J, Møller AM, Afshari A. Thromboelastography (TEG) or rotational thromboelastometry (ROTEM) to monitor haemostatic treatment in bleeding patients: a systematic review with meta-analysis and trial sequential analysis. Anaesthesia 2017;72(04):519-531 